The Spectrum of

Clean Energy Innovation

\title{
"Buried-Anode" Technology Leads to Advanced Lithium Batteries
}

A technology developed at the National Renewable Energy Laboratory (NREL) has sparked a start-up company that has attracted funding from the Advanced Projects Research Agency-Energy (ARPA-E). Planar Energy, Inc. has licensed NREL's "buried-anode" technology and put it to work in solid-state lithium batteries. The company claims its large-format batteries can achieve triple the performance of today's lithium-ion batteries at half the cost, and if so, they could provide a significant boost to the emerging market for electric and plug-in hybrid vehicles.

It all began in 2001, when three NREL researchers took their thin-film expertise from window technology research and applied it to a solid-state, thin-film lithium battery. The researchers knew that lithium batteries tended to degrade quickly because the fragile lithium metal anode was on the top of the battery, where any cracks in the encapsulant could lead to rapid failure.

The team developed the concept of building the battery in reverse order, depositing first the solid-state electrolyte, made of lithium phosphorous oxynitride (LiPON), then the cathode, a metal oxide. Lithium is typically intercalated (chemically trapped) within the cathode material. Placing an initial charge on the battery causes the lithium ions to migrate out of the cathode and through the electrolyte, "plating out" onto the substrate to form a lithium anode that is "buried" below the other layers and protected by them. This "buried-anode" approach allows the battery to be deposited as two thin layers and avoids the need to use bulky encapsulants to protect the battery.

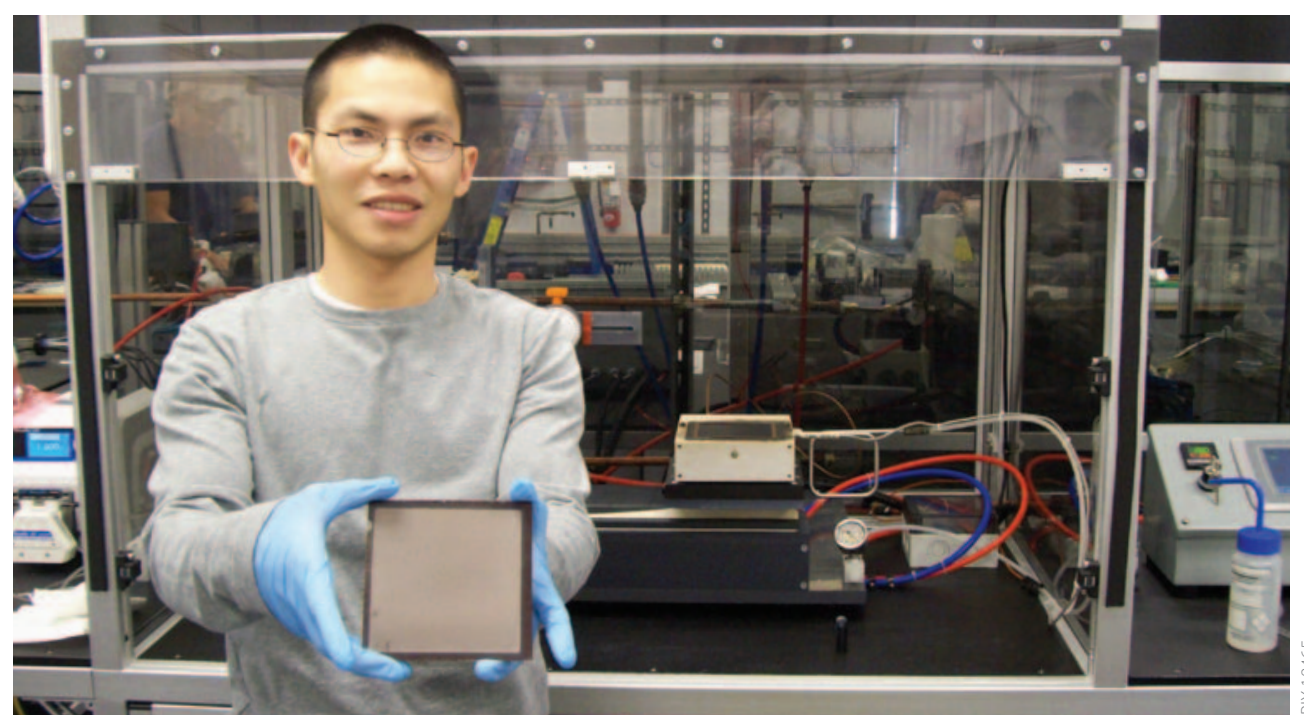

Planar Energy researcher Binh Tran holds a large-format thin-film lithium battery that uses NREL's buried-anode technology. In an electric vehicle, many of these thin-film batteries will be combined to form a high-capacity battery pack. Courtesy of Planar Energy, Inc.

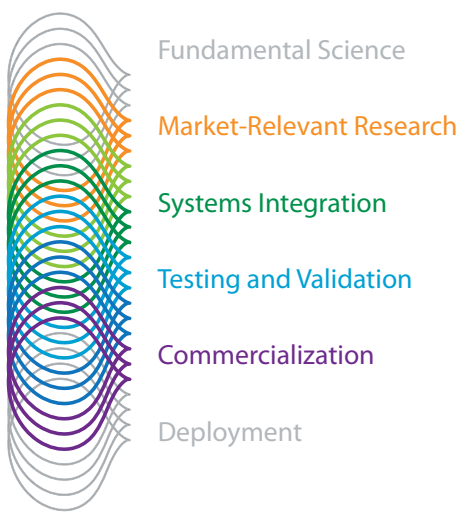

Through deep technical expertise and an unmatched breadth of capabilities, NREL leads an integrated approach across the spectrum of renewable energy innovation. From scientific discovery to accelerating market deployment, NREL works in partnership with private industry to drive the transformation of our nation's energy systems.

This case study illustrates NREL's contributions in MarketRelevant Research through Commercialization.

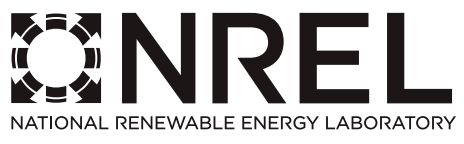

NREL is a national laboratory of the U.S. Department of Energy, Office of Energy Efficiency and Renewable Energy, operated by the Alliance for Sustainable Energy, LLC. 
The NREL researchers built a crude prototype of their invention, proving that it worked, and then filed for a patent, which was granted in 2003. Battelle Ventures then became interested in the technology and provided funding for NREL to further explore its feasibility. That additional research found that the battery technology was very durable and had a high charge capacity, suggesting that it was commercially viable.

Battelle Ventures then chose to spin off the NREL technology, working with an entrepreneur to found Planar Energy, Inc. To help transfer the technology to the company, one of NREL's lead researchers took a sabbatical from the lab to serve as the chief technology officer for the company; he has since become a full-time Planar Energy employee. Planar Energy was able to advance the battery from its crude prototype to a miniaturized, integrated device, which won an R\&D 100 Award in 2009.

The Planar Energy battery is rechargeable, highly compact, and in thin-film form exhibits an extremely long cycle life. Made from only inorganic materials, the battery is immune to the thermal runaway and fires that can occur in lithium-ion batteries, which are the current favorite for deployment in electric vehicles. The Planar Energy battery also tolerates high-temperature environments, allows charging and discharging at high rates, resists self-discharge when not in use, and minimizes degradation during use, even when fully discharged. All of these factors make it a prime candidate for use in electric vehicles.

Another key advantage of the technology is that the layers of the battery can be deposited using high-speed processes, allowing the use of a roll-to-roll manufacturing process similar to the high-speed printing presses that quickly turn rolls of newsprint into newspapers. Planar Energy has developed its own deposition technology called Streaming Protocol for Electroless Electrochemical Deposition, or SPEED, which allows for the manufacture of large-format lithium batteries, thereby opening the door to the electric vehicle market.

The SPEED process uses proprietary water-based precursors that can be applied to large areas of flexible substrates or on top of existing films; these precursors will self-assemble into a new film layer. The film is grown under ambient conditions and at growth rates exceeding 1 micron per minute, a speed suitable for roll-to-roll manufacturing processes.

Planar Energy now intends to produce large-format, solid-state lithium batteries at half the cost and triple the performance of today's lithium-ion batteries. To further the company's development of its large-format battery, Planar Energy has received an award of more than \$4 million from ARPA-E, an agency of the U.S. Department of Energy (DOE).

NREL's involvement with Planar Energy continues through this ARPA-E project. Under a Cooperative Research and Development Agreement (CRADA), NREL is contributing its significant expertise in the development of battery materials, including materials development from theoretical principals; process development and integration; and thermal modeling and testing of complete battery packages. The CRADA has a value of more than $\$ 800,000$ over three years.

Planar Energy is also part of a research and development initiative led by DOE's Oak Ridge National Laboratory to address the energy storage challenges presented by lithium batteries. In addition, Planar Energy won the 2009 World Technology Award under the energy category. This award is selected by the World Technology Network, a global community of peer-elected individuals and organizations in the science and technology world.
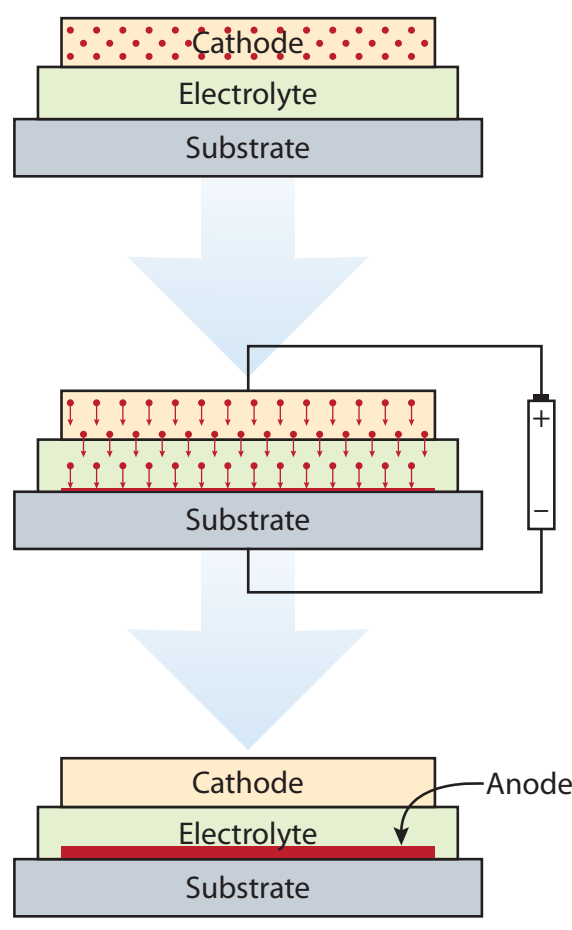

The buried-anode design involves depositing a solidstate electrolyte onto a substrate, such as a metal foil, followed by a cathode containing lithium, such as lithium manganese oxide $\left(\mathrm{LiMn}_{2} \mathrm{O}_{4}\right)$. Applying an initial charging voltage to the battery causes the lithium ions to migrate to the surface of the substrate, where they form a lithium metal anode. The device can also be formed using a cathode material that does not contain lithium by exposing it to a lithium metal vapor and then completing the electrical forming process.

\section{National Renewable Energy Laboratory}

1617 Cole Boulevard

Golden, Colorado 80401-3305

303-275-3000 • www.nrel.gov

NREL is a national laboratory of the U.S. Department of Energy, Office of Energy Efficiency and Renewable Energy, operated by the Alliance for Sustainable Energy, LLC.

NREL/FS-6A42-49149 • February 2011

Printed with a renewable-source ink on paper containing at least $50 \%$ wastepaper, including $10 \%$ post consumer waste. 